\title{
Development of QRS Detection using Short-time Fourier Transform based Technique
}

\author{
Nopadol Uchaipichat \\ Med-Intelligence and Innovation Lab, \\ Faculty of Engineering, \\ Thammasat University, Pathumthani, \\ Thailand
}

\author{
Sakonthawat Inban \\ Med-Intelligence and Innovation Lab, \\ Faculty of Engineering, \\ Thammasat University, Pathumthani, \\ Thailand
}

\begin{abstract}
This paper reports our study in QRS complex detection. The short-time Fourier transform (STFT) was employed in ECG filtering stage. The narrow rectangular window was used to transform ECG signals into time-frequency domain. The temporal information at $45 \mathrm{~Hz}$ from spectrogram was analyzed for detecting QRS locations. The automated thresholding combined with local maxima finding method was modified to find the QRS location. The data used in this study is MIT-BIH Arrhythmia database. As the results, our proposed technique achieved the detection rate better than $99 \%$ and fail ratio was $1.3 \%$.
\end{abstract}

\section{Keywords}

QRS detection, Electrocardiogram, Shot-time Fourier Transform

\section{INTRODUCTION}

Currently, the number of victims of sudden cardiac arrest increases every year. The electrocardiogram (ECG) represents electrical activities of heart that is generally used in contemporary medicine. The main morphology of ECG consists of $\mathrm{P}$ wave, QRS complex, and $\mathrm{T}$ wave for each cycle of cardiovascular. This information of ECG is very useful for cardiologist in diagnosis of abnormalities of the heart [1]. In the measurement of ECG, the signal sometimes includes some unwanted information such as power-line interference, ECG baseline wander, etc. These interferences may effect on the diagnostic. The finding of QRS complex is usually investigated for beat detection since the QRS complex is the dominant component of each normal ECG cycle. Therefore, several research groups have developed many methods to find the location of QRS complex. For instance Chen et al. combined discrete wavelet transform and adaptive thresholding method to detect QRS complexes [2]. Darrington proposed the fast method with minimal pre-process for real time QRS detection [3]. In addition to Mehta et al. applied support vector machine (SVM) and K-means algorithms to detection QRS complexes [4,5]. Therefore, in this study we have proposed novel technique to detect the QRS complex of ECG surface. The normal ECG recordings from MIT-BIH Arrhythmia database were studied in our investigation. The narrow window of short-time Fourier transform (STFT) was employed to remove unwanted information such as $\mathrm{P}$ wave, $\mathrm{T}$ wave and noises. The adaptive thresholding method was proposed to detect the QRS complex.

\section{MATERIALS AND METOHODOLOGY}

\subsection{Data Study}

The datasets used in this study were obtained from MIT-BIH Arrhythmia database available in Physionet website [6]. The data contain 48 half-hour ECG recordings obtained from 47 subjects. Each recording was collected at $360 \mathrm{~Hz}$ of sampling rate with 11bit resolution over a $10 \mathrm{mV}$ range. The dataset also includes annotation files evaluated from cardiologist for indicating the information of ECG traces. Most studied ECG signals are from modified limb lead II (MLII) except modified lead V5 was used for record 102 and 104. The dataset totally contains 116,137 beats.

\subsection{ECG Filtering and QRS Complex Detection Method}

This section describes our proposed technique to remove $\mathrm{P}$ wave and $\mathrm{T}$ wave. In this paper the short time Fourier transform (STFT) was applied in ECG processing. The STFT is the technique for non-stationary signal analysis that transforms signal information from time domain into time-frequency domain. The main concept of the STFT is to consider a nonstationary signal as a stationary signal over short periods of time within a window function $[7,8]$. The computation of STFT can be defined as equation (1).

$$
T(f, \tau)=\int_{-\infty}^{\infty}[x(t) w(t-\tau)] e^{-j 2 \pi f t} d t
$$

where $w(t-\tau)$ is the window function. From equation (1) the STFT maps signal $x(t)$ into two-dimensional function in time, $\tau$, and frequency, $f$. The energy surface distribution of STFT called spectrogram can be computed from equation (2)

$$
E(f, \tau)=|T(f, \tau)|^{2}
$$

The rectangular window was used in this study. The narrow window width of 16-point was used because the high resolution in time is required to detect QRS complex. Figure 1 shows the example of ECG time series and its corresponding STFT spectrogram. For this example the simulated noise was added into original ECG as defined in equation (3).

$$
x_{n}(t)=x(t)+n(t)
$$


where $x(t)$ presents original ECG signal and $n(t)$ presents function of simulated noise. The adding noise contains both low and high frequency of interference. Equation (4) expresses the function of simulated noise which contains noise of $0.5 \mathrm{~Hz}$ and $50 \mathrm{~Hz}$.

$$
n(t)=100 \cos (\pi t)+50 \cos (100 \pi t)
$$
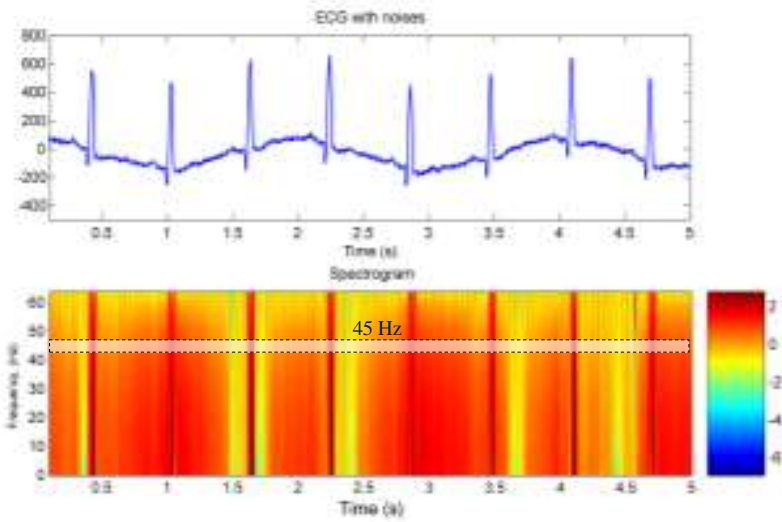

Figure 1. The STFT of example ECG

From spectrogram in figure 1, the highlighted box illustrates the temporal information at $45 \mathrm{~Hz}$ that was used for the finding of QRS location. The STFT temporal information at $45 \mathrm{~Hz}$ can be defined as equation (5).

$$
E(\tau)=\log (E(45, \tau))
$$

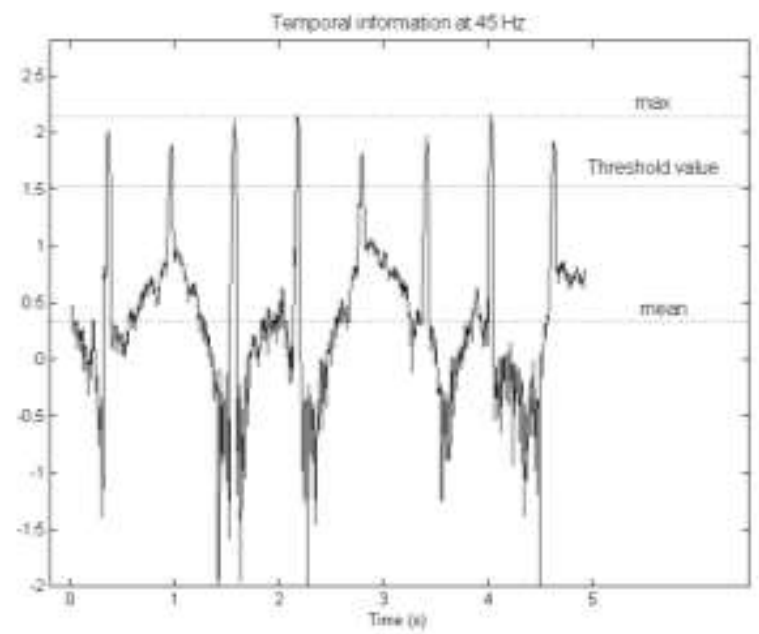

Figure 2. The temporal information of $45 \mathrm{~Hz}$

Figure 2 shows the STFT temporal information of ECG at $45 \mathrm{HZ}$. In thresholding stage, the threshold value was determined by using simple adaptive thresholding method that can be defined as equation (6)

$$
T h \_v a l=M e a n+\frac{2}{3}(\text { Max }- \text { Mean })
$$

where Mean and Max presents the average value and maximum value of $E(\tau)$ in equation (5) respectively. The thresholding condition can be described in equation (7). Figure 3 shows the result of thresholding stage.

$$
E(\tau)= \begin{cases}E(\tau) & ; E(\tau) \geq T h_{-} \text {val } \\ \text { Mean } & ; E(\tau)<T h_{-} \text {val }\end{cases}
$$

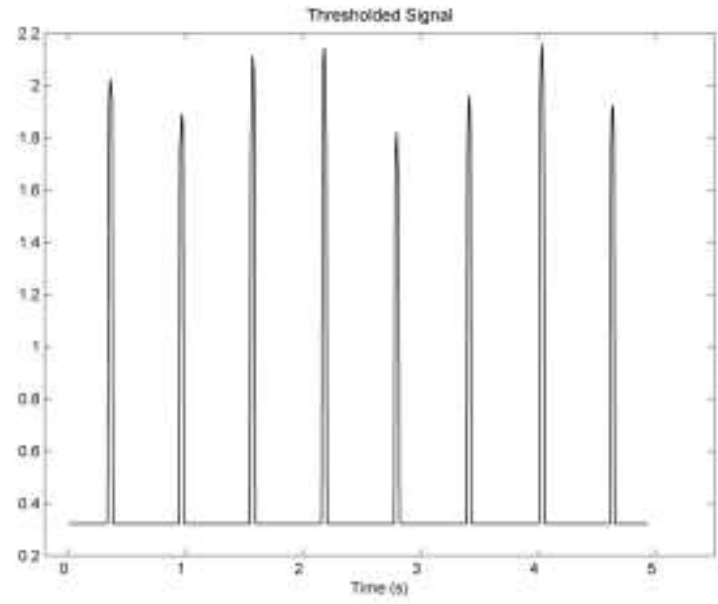

Figure 3. The thresholded information

The locations of QRS complexes were then evaluated from the position of local maxima. In this binarization stage the value of thresholded signal at position of local maxima were set to one and others were set to zero. The binary signal can be illustrated in figure 4. It can be seen that eight positions of QRS complexes were detected for this example. Comparing to ECG trace this method can detect the position of QRS complexes correctly.

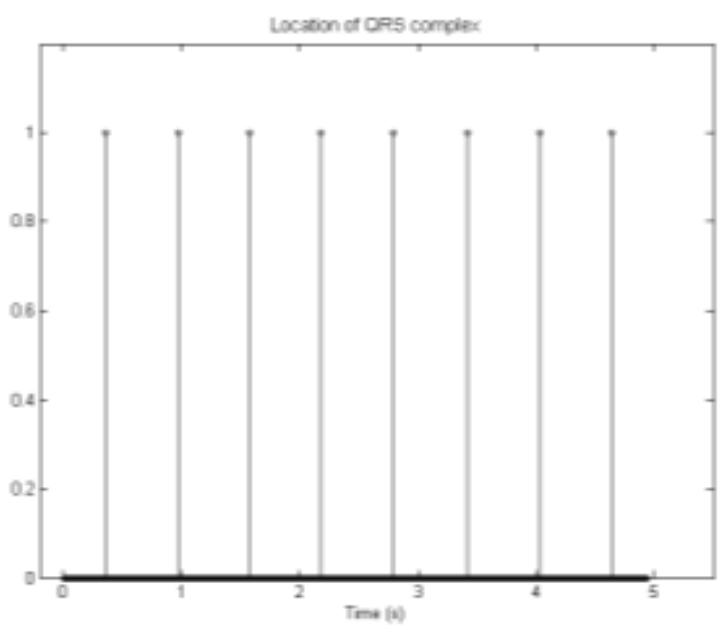

Figure 4. The signal after binarization stage 
Table 1. The experimental results

\begin{tabular}{|c|c|c|c|c|c|c|c|}
\hline File & Beats & TP & FP & FN & Sens & Spec & $\begin{array}{l}\text { Fail } \\
\text { Ratio }\end{array}$ \\
\hline 100 & 2273 & 2273 & 0 & 0 & 1.000 & 1.000 & 0.000 \\
\hline 101 & 1865 & 1863 & 8 & 2 & 0.999 & 0.996 & 0.005 \\
\hline 102 & 2187 & 2186 & 0 & 1 & 1.000 & 1.000 & 0.000 \\
\hline 103 & 2084 & 2084 & 0 & 0 & 1.000 & 1.000 & 0.000 \\
\hline 104 & 2229 & 2205 & 21 & 24 & 0.989 & 0.991 & 0.020 \\
\hline 105 & 2572 & 2526 & 35 & 46 & 0.982 & 0.986 & 0.031 \\
\hline 106 & 2027 & 2016 & 1 & 11 & 0.995 & 1.000 & 0.006 \\
\hline 107 & 2137 & 2131 & 1 & 6 & 0.997 & 1.000 & 0.003 \\
\hline 108 & 1763 & 1722 & 53 & 41 & 0.977 & 0.970 & 0.053 \\
\hline 109 & 2532 & 2531 & 0 & 1 & 1.000 & 1.000 & 0.000 \\
\hline 111 & 2124 & 2121 & 6 & 3 & 0.999 & 0.997 & 0.004 \\
\hline 112 & 2539 & 2538 & 0 & 1 & 1.000 & 1.000 & 0.000 \\
\hline 113 & 1795 & 1795 & 0 & 0 & 1.000 & 1.000 & 0.000 \\
\hline 114 & 1880 & 1871 & 4 & 8 & 0.996 & 0.998 & 0.006 \\
\hline 115 & 1953 & 1953 & 0 & 0 & 1.000 & 1.000 & 0.000 \\
\hline 116 & 2412 & 2397 & 6 & 15 & 0.994 & 0.998 & 0.009 \\
\hline 117 & 1535 & 1534 & 54 & 1 & 0.999 & 0.966 & 0.036 \\
\hline 118 & 2278 & 2277 & 3 & 1 & 1.000 & 0.999 & 0.002 \\
\hline 119 & 1987 & 1987 & 1 & 0 & 1.000 & 0.999 & 0.001 \\
\hline 121 & 1863 & 1862 & 3 & 1 & 0.999 & 0.998 & 0.002 \\
\hline 122 & 2476 & 2476 & 0 & 0 & 1.000 & 1.000 & 0.000 \\
\hline 123 & 1518 & 1518 & 3 & 0 & 1.000 & 0.998 & 0.002 \\
\hline 124 & 1619 & 1618 & 1 & 1 & 0.999 & 0.999 & 0.001 \\
\hline 200 & 2601 & 2580 & 25 & 21 & 0.992 & 0.990 & 0.018 \\
\hline 201 & 1963 & 1961 & 112 & 2 & 0.999 & 0.946 & 0.058 \\
\hline 202 & 2136 & 2135 & 1 & 1 & 1.000 & 1.000 & 0.001 \\
\hline 203 & 2980 & 2698 & 29 & 282 & 0.905 & 0.989 & 0.104 \\
\hline 205 & 2656 & 1639 & 0 & 17 & 0.990 & 1.000 & 0.006 \\
\hline 207 & 2344 & 2022 & 11 & 322 & 0.863 & 0.995 & 0.142 \\
\hline 208 & 2955 & 2929 & 3 & 26 & 0.991 & 0.999 & 0.010 \\
\hline 209 & 3005 & 3004 & 1 & 1 & 1.000 & 1.000 & 0.001 \\
\hline 210 & 2650 & 2634 & 5 & 16 & 0.994 & 0.998 & 0.008 \\
\hline 212 & 2748 & 2748 & 1 & 0 & 1.000 & 1.000 & 0.000 \\
\hline 213 & 3251 & 3248 & 0 & 3 & 0.999 & 1.000 & 0.001 \\
\hline 214 & 2262 & 2258 & 4 & 4 & 0.998 & 0.998 & 0.004 \\
\hline 215 & 3363 & 3272 & 1 & 91 & 0.973 & 1.000 & 0.027 \\
\hline 217 & 2208 & 2204 & 1 & 4 & 0.998 & 1.000 & 0.002 \\
\hline 219 & 2154 & 2154 & 0 & 0 & 1.000 & 1.000 & 0.000 \\
\hline 220 & 2048 & 2048 & 0 & 0 & 1.000 & 1.000 & 0.000 \\
\hline 221 & 2427 & 2426 & 1 & 1 & 1.000 & 1.000 & 0.001 \\
\hline 222 & 2483 & 2481 & 2 & 2 & 0.999 & 0.999 & 0.002 \\
\hline 223 & 2605 & 2604 & 0 & 1 & 1.000 & 1.000 & 0.000 \\
\hline 228 & 2056 & 1996 & 28 & 57 & 0.972 & 0.986 & 0.041 \\
\hline 230 & 2256 & 2255 & 2 & 1 & 1.000 & 0.999 & 0.001 \\
\hline 231 & 1571 & 1571 & 0 & 0 & 1.000 & 1.000 & 0.000 \\
\hline 232 & 1780 & 1779 & 0 & 1 & 0.999 & 1.000 & 0.001 \\
\hline 233 & 3079 & 3071 & 0 & 8 & 0.997 & 1.000 & 0.003 \\
\hline 234 & 2753 & 2753 & 1 & 0 & 1.000 & 1.000 & 0.000 \\
\hline & & & \multicolumn{2}{|c|}{ Average } & 0.991 & 0.996 & 0.013 \\
\hline
\end{tabular}

\section{EXPERIMENTAL RESULTS}

From our experiment, each position of QRS complex obtained from our proposed method was compared to the given annotation available in MIT-BIH Arrhythmia database. The accepted tolerance was set to $200 \mathrm{~ms}$. The performance of QRS detection can be measured from sensitivity and specificity [3]. The sensitivity and specificity can be defined as equation (8) and (9) in respectively.

$$
\begin{aligned}
& \text { Sens }=\frac{T P}{T P+F N} \\
& \text { Spec }=\frac{T P}{T P+F P}
\end{aligned}
$$

The true positive (TP) presents number of detected beats from actual beats. The false negative (FN) presents number of undetected beats from actual beats. The false positive (FP) presents number of detected beat from non beats. Table 1 presents our experimental results in QRS detection with 48 studied ECG files. The proposed technique achieved the performance in beat detection at $99.1 \%$ sensitivity and $99.6 \%$ specificity. There are 107,953 beats that can be detected from 109,982 actual beats. The number of wrong detection is 1,452 beats that equals to $1.3 \%$ of fail ratio.

Table 2. Comparison of results with other methods

\begin{tabular}{|l|c|c|c|c|}
\hline & Sens & Spec & $\begin{array}{c}\text { Detection } \\
\text { rate }\end{array}$ & $\begin{array}{c}\text { Fail } \\
\text { Ratio }\end{array}$ \\
\hline Proposed Method & $99.10 \%$ & $99.60 \%$ & $99.10 \%$ & $1.30 \%$ \\
\hline Darrington [3] & $99.00 \%$ & $99.20 \%$ & $99.00 \%$ & $1.70 \%$ \\
\hline Chen et al. [2] & $99.55 \%$ & $99.49 \%$ & $99.49 \%$ & $0.96 \%$ \\
\hline Mehta et al. [4] & $\mathrm{n} / \mathrm{a}$ & $\mathrm{n} / \mathrm{a}$ & $98.63 \%$ & $\mathrm{n} / \mathrm{a}$ \\
\hline Mehta et al. [5] & $\mathrm{n} / \mathrm{a}$ & $\mathrm{n} / \mathrm{a}$ & $99.30 \%$ & $\mathrm{n} / \mathrm{a}$ \\
\hline
\end{tabular}

\section{DISCUSSION AND CONCLUSION}

This paper proposed the STFT technique for beat detection. The temporal information at $45 \mathrm{~Hz}$ combined with adaptive thresholding method was employed for finding the $\mathrm{R}$ peak. The 48 cases with 109,982 beats were tested in our experiment. The result is comparable to other works which investigated the same topic. The comparison can be presented in table 2 . It can be seen that the detection rate is general used to measure the detection performance. The detection rate can be determined from minimum value among sensitivity and specificity [2]. As the result the proposed method achieved detection rate at $99.10 \%$ that is nearly on par with other popular methods. However, the results done by Mehta et al were obtained from different database [4,5]. This paper shows that the STFT technique has potential to improve the performance in QRS complex detection. The advanced methods, such as artificial neural networks and fuzzy logic, are suggested to be employed in the stage of decision making. The limitation of this investigation is that time complexity has not been studied in this paper. 


\section{ACKOWLEDGEMENT}

This research is financially supported by the Thailand Research Fund (TRF), contract number. MRG5380184.

\section{REFERENCES}

[1] Houghton, A.R. and Gray, D., 1997, Making Sense of the ECG, A hands-on guide, ARNOLD.

[2] Chen, S.-W., Chen, H.-C., Chan, H.-L., 2006, A real-time QRS detection method based on moving-averaging incorporating with wavelet denoising, Computer Methods and Programs in Biomedicine, 82 (2006), 187-195.

[3] Darrington, J., 2006, Towards real time QRS detection: A fast method using minimal pre-processing, Biomedical Signal Processing and Control, 1 (2006), 169-176.

[4] Mehta, S.S., Shete, D.A., Lingayat, N.S., Chouhan, V.S., 2010, IRBM, 31 (2010), 48-54.
[5] Mehta, S.S., Lingayat, N.S., 2008, SVM-based algorithm for recognition of QRS complexes in electrocardiogram, IRBM, 29 (2008), 310-317.

[6] Goldberger, A.L., Amaral, L.A.N., Glass, L., Hausdorff, J.M., Ivanov, P.Ch., Mark, R.G., Mietus, J.E., Moody, G.B., Peng, C-K., Stanley, H.E., 2000, PhysioBank, PhysioToolkit, and PhysioNet: components of a new research resource for complex physiologic signals, Circulation, 101 (2000), E215-220.

[7] Gade, S., Gram-Hansen, K., 1997, The analysis of nonstationary signals, Sound and Vibration, 31(1997), 4046.

[8] Rioul, O., Vetterli, M., 1991, Wavelets and signal processing , IEEE Signal Processing Magazine, 8 (1991) 14 38. 